\title{
Dentes supranumerários impactados em região de nervo mentoniano: relato de caso
}

\author{
Supernumerary impacted teeth in the mental nerve region: case report \\ Dientes supernumerarios impactados en la región del nervio mentoniano: reporte de caso
}

Recebido: 26/11/2021 | Revisado: 02/12/2021 | Aceito: 04/12/2021 | Publicado: 14/12/2021

Thaisa Reis de Carvalho Sampaio

ORCID: https://orcid.org/0000-0002-4345-3662 Universidade de Pernambuco, Brasil E-mail: thaisa.sampaio@upe.br

Danilo Barbosa Ferraz

ORCID: https://orcid.org/0000-0002-7257-0841 Centro Universitário Brasileiro, Brasil E-mail: nilobarbosa11@ @otmail.com

Emilyn Kétilin Muniz Silva

ORCID: https://orcid.org/0000-0003-1990-7529 Centro Universitário Brasileiro, Brasil E-mail: emilynkeetilin@gmail.com

Giulianna de Fátima Silva de Arruda ORCID: https://orcid.org/0000-0002-5479-7006 Centro Universitário Brasileiro, Brasil E-mail: giuliannaarruda@gmail.com

Gleicielly Lira da Silva

ORCID: https://orcid.org/0000-0002-6716-411X Centro Universitário Brasileiro, Brasil E-mail: gleicielly.gl@gmail.com

Maria Emanuela Letícia da Silva ORCID: https://orcid.org/0000-0002-7159-1165 Centro Universitário Brasileiro, Brasil E-mail: emanuelaleticia@hotmail.com

Emanuel Savio de Souza Andrade ORCID: https://orcid.org/0000-0003-2165-4217 Universidade de Pernambuco, Brasil E-mail: emanuel.savio@upe.br

\begin{abstract}
Resumo
Introdução: Durante o desenvolvimento dentário, distúrbios podem acometer o germe em desenvolvimento, provocando alterações em número, formato, tamanho, posição e estrutura. A ocorrência de dentes supranumerários representa uma anormalidade sendo mais predominante em dentição permanente. Essa ocorrência, quando relacionada a região de prémolares, diferenciam-se das demais possuindo uma maior frequência, e este fato pode ser explicado por serem um dos últimos dentes a irromper na arcada dentária. Um bom exame clínico, associado a solicitação de exames complementares são fundamentais para um diagnóstico precoce e promissor. Os exames de imagens tornam-se indispensáveis para determinar a localização do elemento extranumerário, além de visualizar possíveis proximidades com estruturas nobres adjacentes. Objetivo: Relatar um caso clínico de exodontia de dentes supranumerários e impactados com finalidade ortodôntica assim como evidenciar a importância de uma anamnese bem detalhada. Relato de caso: Paciente buscou os serviços de uma clínica odontológica particular com indicação ortodôntica para remoção de dentes supranumerários. $\mathrm{O}$ tratamento indicado ao paciente foi a exodontia dos elementos extranumerários, para que ocorresse a movimentação ortodôntica de maneira correta. Conclusão: A remoção dos elementos hiperdônticos em sua grande maioria torna-se indispensável a fim de evitar maiores prejuízos ao paciente, e para que esse procedimento seja realizado de maneira segura, deve-se sempre realizar um exame clínico com riqueza de detalhes combinado com a solicitação dos exames complementares de imagem, com a finalidade de melhor avaliar as estruturas adjacentes ao elemento anômalo.
\end{abstract}

Palavras-chave: Extração dentária; Cirurgia bucal; Dente supranumerário; Dente impactado.

\begin{abstract}
Introduction: During tooth development, disorders can affect the developing germ, causing changes in number, shape, size, position and structure. The occurrence of supernumerary teeth represents an abnormality that is more prevalent in permanent dentition. This occurrence, when related to the premolar region, differs from the others, having a higher frequency, and this fact can be explained by the fact that they are one of the last teeth to erupt in the dental arch. A good clinical examination, associated with the request of complementary exams are fundamental for an early and promising diagnosis. Imaging exams become indispensable to determine the location of the extranumerary element, besides
\end{abstract}


visualizing possible proximities with adjacent noble structures. Objective: To report a clinical case of extraction of supernumerary and impacted teeth for orthodontic purposes, and to highlight the importance of a detailed anamnesis. Case Report: A patient sought the services of a private dental clinic with an orthodontic indication for removal of supernumerary teeth. The treatment indicated to the patient was the exodontia of the supernumerary elements so that the orthodontic movement could occur in a correct manner. Conclusion: The removal of hyperdontic elements in the vast majority of cases is essential in order to avoid further harm to the patient. For this procedure to be safely performed, a detailed clinical examination should always be carried out, combined with a request for complementary imaging tests, in order to better evaluate the structures adjacent to the anomalous element.

Keywords: Tooth extraction; Surgery, oral; Tooth, supernumerary; Tooth, impacted.

\section{Resumen}

Introducción: Durante el desarrollo dental, los trastornos pueden afectar al germen en desarrollo, provocando cambios en número, forma, tamaño, posición y estructura. La aparición de dientes supernumerarios representa una anomalía que es más prevalente en la dentición permanente. Esta ocurrencia, cuando se relaciona con la región premolar, se diferencia de las demás, teniendo una mayor frecuencia, y este hecho puede explicarse por el hecho de que son uno de los últimos dientes en erupcionar en la arcada dentaria. Un buen examen clínico, asociado con la solicitud de pruebas adicionales, es esencial para un diagnóstico temprano y prometedor. Los exámenes de imagen son esenciales para determinar la ubicación del elemento supernumerario, además de visualizar posibles proximidades con estructuras nobles adyacentes. Objetivo: Informar un caso clínico de extracción de dientes supernumerarios e impactados con fines de ortodoncia, así como resaltar la importancia de una anamnesis detallada. Caso clínico: Paciente solicitó los servicios de una clínica dental privada con indicación de ortodoncia para la extracción de dientes supernumerarios. El tratamiento indicado para el paciente fue la extracción de los elementos extranumerarios, para que el movimiento ortodóncico pudiera realizarse correctamente. Conclusión: La eliminación de la mayoría de los elementos hiperdónticos es esencial para evitar un daño mayor al paciente, y para que este procedimiento se realice de manera segura, un examen clínico con ricos detalles combinado con la solicitud de exámenes de imagen adicionales, con el fin de evaluar mejor la estructuras adyacentes al elemento anómalo.

Palabras clave: Extracción dental; Cirugía bucal; Diente supernumerario; Diente impactado.

\section{Introdução}

A hiperdontia ou dente supranumerário é uma anormalidade do desenvolvimento dentário, sendo definida como a presença de elementos que excedem a quantidade normal de dentes decíduos ou permanentes em boca (Laganà et al., 2011).

Não existe um consenso entre os pesquisadores sobre a etiologia dos dentes supranumerários, existindo ainda diversas teorias que explicam a ocorrência do evento. Acredita-se que a formação desses dentes advém do crescimento de remanescentes da lâmina dentária; da atividade anormal das células da lâmina dentária, que resulta na produção um número anormal de germes; ou ainda, podendo ser resultado da dicotomia de um germe em desenvolvimento (Stringhini Junior et al., 2015).

O termo impactação é definido como a falta de erupção de um dente dentro dos limites fisiológicos de tempo do processo de erupção normal. Acontece uma falha na erupção do dente permanente ou decíduo, que está com rizogênese completa ou não, todavia não consegue irromper por conta da impactação causada no mesmo (Dalessandri et al., 2017; Mohammed et al., 2020).

A etiologia das impactações ainda é um ponto bastante divergente entre as pesquisas já realizadas, inúmeros autores descrevem distintas condições relevantes que levam um dente a impactar, sendo eles: origem genética, obstrução dos incisivos laterais, deficiência maxilar e distúrbios relacionados ao folículo dentário (Mohammed et al., 2020; Lima et al., 2019; Ngan et al., 2005; Franco et al., 2005).

A taxa de acometimento dos dentes supranumerários descritos na literatura varia entre 0,1 à $3,8 \%$. A localização de maior ocorrência é na maxila, especificamente a região dos incisivos centrais superiores, seguida por regiões como incisivos laterais superiores, pré-molares inferiores e região localizada a distal de terceiros molares (Coelho et al., 2011).

A literatura relata predileção do sexo feminino para a prevalência da impactação dentária, além de uma maior predominância de pessoas entre a segunda e terceira década de vida (Kaczor-Urbanowicz et al., 2016; Alves et al., 2014).

Os dentes supranumerários são classificados como distomolar (quarto molar), mesiodens (quando surgem na linha média, entre os incisivos centrais superiores), paramolar (com localização lingual ou vestibular a um molar). Os supranumerários 
são divididos em tipos: Suplementar (tamanhos e formas normais) e rudimentar (forma anormal e tamanho menor) (Khalaf et al., 2018; Scully et al., 2020; Oliveira et al., 2015).

No tocante ao diagnóstico de pré-molares supranumerários, os métodos radiográficos convencionais, como as radiografias panorâmicas, apresentam limitações, uma vez que fornecem ao cirurgião uma imagem bidimensional, que culmina a sobreposição de estruturas, mascarando a real posição do dente. Técnicas de imagem como a tomografia computadorizada de feixe cônico (TCFC) com vantagens de baixa radiação, varredura rápida de imagens com dados radiográficos e volumétricos 3D, têm sido utilizadas para diagnóstico. A literatura afirma a importância do diagnóstico clínico e radiográfico da hiperdontia, evitando prognósticos negativos para o paciente, tratando esses casos de forma mais eficiente e segura (Franco et al., 2006; Dalessandri et al., 2016; Mohammed et al., 2020).

A presença de dentes supranumerários na arcada dentária pode causar problemas, dependendo de sua localização. Eles podem causar a impactação de dentes permanentes, reabsorção radicular, impedir a movimentação ortodôntica, estimular a formação de cistos odontogênicos (Hupp et al., 2009).

O tratamento para hiperdontia dos pré-molares geralmente é cirúrgico, com a priorização da exodontia atraumática, a fim de diminuir possíveis traumas de natureza operatória e amenizar a perda óssea alveolar após a extração do dente. O tratamento deve ser de caráter multidisciplinar, já que envolve diferentes áreas da Odontologia como Cirurgia, Ortodontia, Periodontia e Radiologia. Diante do contexto exposto, o presente estudo tem por objetivo relatar um caso de exodontia de pré-molares mandibulares supranumerários.

\section{Relato de Caso}

Paciente N.L.C.C, sexo feminino, 16 anos de idade, melanoderma, compareceu a uma clínica particular na cidade de Recife/PE com indicação ortodôntica para remoção de dentes supranumerários. Ao exame clínico, o responsável pela paciente relata que a mesma não apresentava nenhuma doença sistêmica. Foi solicitada uma tomografia computadorizada de feixe cônico (TCFC), onde constatou a presença de pré-molares supranumerários impactados em região de nervo mentoniano, com uma maior proximidade da tábua óssea mandibular lingual (Figura 1).

Figura 1. Tomografia computadorizada de feixe cônico.

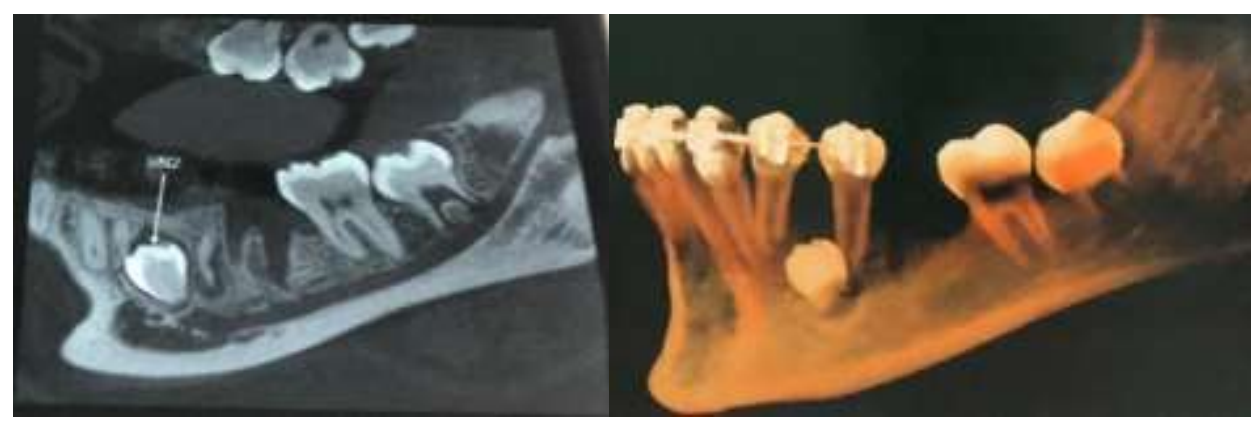

Fonte: Autores.

O procedimento cirúrgico foi realizado sob anestesia local, com bloqueio dos nervos alveolar inferior, mentoniano e lingual utilizando-se com o anestésico local cloridrato de mepivacaína a 2\% com adrenalina 1:100.000 (Nova DFL, São Paulo, Brasil). Um retalho lingual do tipo envelope foi confeccionado do dente 35 ao dente 45 e realizou-se descolamento mucoperiosteal (Figura 2). 
Figura 2. Retalho tipo envelope com descolamento mucoperiosteral em região lingual.

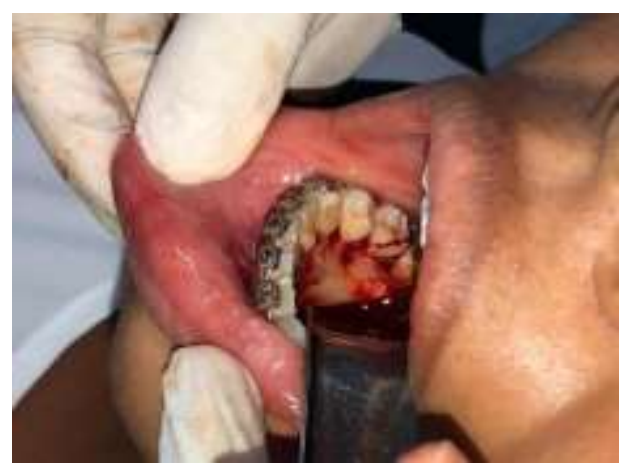

Fonte: Autores.

Foi realizada osteotomia da tábua óssea lingual e odontosecção nos dentes com auxílio da caneta de alta rotação e broca cirúrgica ${ }^{\circ} 702$ (JET, São Paulo, Brasil) sob irrigação constante com soro fisiológico e exodontia dos dentes inclusos e remoção do capuz coronário (Figura 3).

Figura 3. Realização da osteotomia para exodontia dos elementos supranumerários.

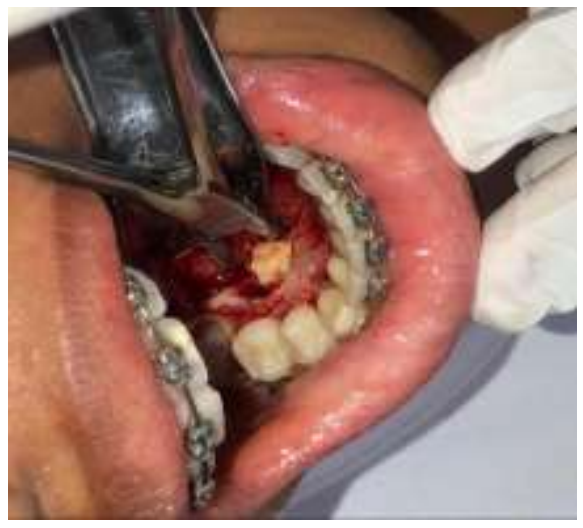

Fonte: Autores.

Em seguida, fez-se a regularização óssea, lavagem abundante da ferida com soro fisiológico e sutura com fio de seda 30 (Ethicon, São Paulo, Brasil) por primeira intenção (Figura 4).

Figura 4. Sutura realizada com fio de seda 3-0.

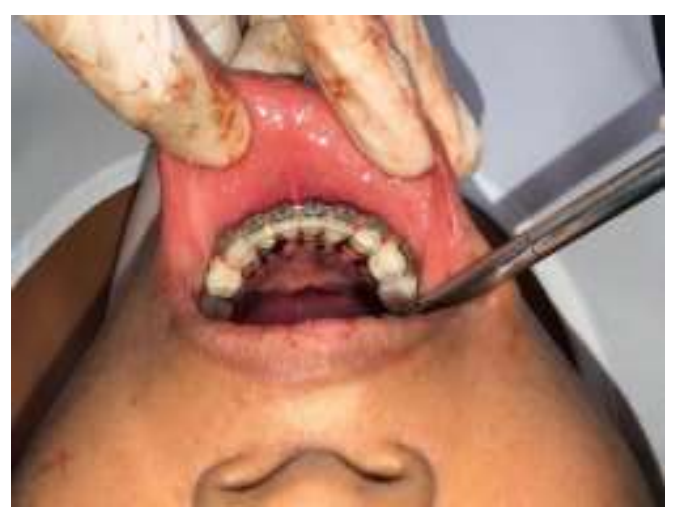

Fonte: Autores. 


\section{Discussão}

A impactação dos pré-molares permanentes é um fato recorrente na vivência clínica odontológica. Por ser um dos últimos dentes a serem erupcionados na arcada dentária permanente, esse fato pode explicar a ocorrência da tal impactação.

Um bom exame clínico e uma história médica cuidadosa são os elementos-chave para um bom planejamento e determinação do melhor método para um bom prognóstico clínico, assim como o esclarecimento do tratamento preconizado ao paciente (Marsi, et al., 2009; Luz, et al., 2014).

Vuchkova et al. (2010) e Stringhini Junior et al. (2015) em seus estudos descrevem dois principais tratamentos, a terapêutica conservadora e a não conservadora. $\mathrm{O}$ tratamento conservador consiste na exposição cirúrgica do dente para posterior tracionamento. Entretanto, o não conservador consiste na remoção dos dentes cirurgicamente.

Os exames imaginológicos são de fundamental importância para entender a complexidade da região a ser operada, sendo utilizados na rotina da Cirurgia Bucomaxilofacial como coadjuvante para o exame físico e consequente sucesso no tratamento do paciente.

A radiografia panorâmica é uma técnica muito eficaz quando se trata de diagnóstico preciso em relação aos dentes não irrompidos, além disso produz uma única imagem radiográfica das estruturas faciais que inclui ambos os arcos dentários, maxilar e mandibular, e suas estruturas de suporte. $\mathrm{O}$ exame também proporciona a visualização do canino em relação às demais estruturas, como linha média e plano oclusal, por exemplo. Entretanto, por ser um exame bidimensional, ocasiona sobreposições de imagens, além de não possuir um grande nível de detalhes (Fox et al., 1995; White \& Pharoah, 2015).

A Tomografia Computadorizada de Feixe Cônico se sobressai dentre os outros exames imaginológicos por possibilitar a reprodução de uma imagem tridimensional das estruturas do corpo humano, favorecendo a localização de estruturas nobres e dentes que nos exames convencionais não seria possível a visualização. eventualmente contribuindo para o sucesso do tratamento no caso apresentado (White \& Pharoah, 2015; Stuani et al., 2016). No caso apresentado, solicitou-se uma tomografia de feixe cônico, dando uma melhor visualização para planejamento adequado das áreas a serem operadas.

Assim como diversos procedimentos cirúrgicos existem possibilidades de complicações. Dentre as complicações podese ressaltar as lesões de estruturas nobres, laceração do retalho mucoso, esgarçamento ou abrasão, fratura do processo alveolar, lesão de raízes dos dentes permanentes adjacentes, lesões a estruturas nervosas regionais, infecção, deiscência de feridas, alvéolo seco e parestesia (Hupp et al., 2009; Benevides et al., 2018). No presente caso, não houve nenhuma intercorrência transoperatória ou pós-operatória.

Uma conduta multidisciplinar é fundamental para atender às necessidades da maioria dos pacientes, de modo que

exista um atendimento assistencial e coordenado entre os dentistas generalistas e as especialidades de maneira que exista uma otimização do tratamento (Franco et al., 2006; Hupp et al., 2009; Ferri et al., 2015). Foi crucial no caso em questão a atuação do ortodontista para prévia avaliação ortodôntica, porém ao realizar o exame tomográfico, constatou-se impossibilidade do tracionamento dos dentes, por isso foi necessário a adoção do Cirurgião Bucomaxilofacial na equipe, para a execução da exodontia dos dentes.

\section{Conclusão}

É necessário estar atento quanto a presença de dentes supranumerários na arcada dentária, principalmente quando o paciente irá iniciar algum tratamento ortodôntico, a fim de evitar problemas maiores. Os exames de imagem são fundamentais para diagnóstico e planejamento do tratamento desta condição. Quando indicada a exodontia, a mesma deve ser realizada com cautela para evitar danos a estruturas importantes. 


\section{Referências}

Alves, E. P., Montagner, A. F., Antoniazzi, S. P., \& Oliveira, L. F. D. D. (2014). Prevalence and position of impacted maxillary canines and their relation with root resorption. RFOUPF, 19(2), 180-184.

Benevides, R. R., Valadas, L. A. R., Diógenes, E., Rodrigues, E., \& Furtado, J. (2018). Parestesia do nervo alveolar inferior após exodontia de terceiros molares inferiores: da prevenção ao tratamento. Rev. FullDent. Sci, 9(35), 66-71.

C. White, S., \& J. Pharoah, M. (2015). Radiologia Oral: princípios e interpretações. Elsevier, 7, 392.

Coelho, A., Macho, V., Andrade, D., Macedo, P., \& Areias, C. (2011). Prevalência e distribuicão de dentes supranumerários numa populacão pediátrica-Um estudo radiográfico. Revista Portuguesa de Estomatología, Medicina Dentária e Cirugia Maxilofacial, 52(4), $189-192$.

Dalessandri, D., Parrini, S., Rubiano, R., Gallone, D., \& Migliorati, M. (2017). Incidência, etiologia e tratamento de caninos mandibulares impactados e transmigrantes: uma revisão sistemática. European Journal of Orthodontics , 39 (2), 161-169.

Ferri, E. A., Moresca, R., Braga, J. V., Morosini, I. D. A. C., Nerone, L. A., \& Oliveira, W. L. D. (2015). Tratamento multidisciplinar de incisivos centrais superiores impactados. Ortho Sci., Orthod. sci. pract, 8(30), 217-225.

Fox, N. .A, Fletcher, GA, \& Horner, K. (1995). Localização de caninos superiores por meio de tomografia panorâmica dentária. British Dental Journal , 179 (11), 416-420.

Franco, A. D. A., Paixão, G. B., Cevidanes, L. H. S., \& Chaves Júnior, C. M. (2006). Abordagem multidisciplinar dos caninos superiores permanentes impactados. Ortodontia, 350-359.

Hupp, J. R., Ellis, E. T., \& MR, C. (2015). oral e maxilofacial contemporânea (p. 980). Elsevier.

Kaczor-Urbanowicz, K., Zadurska, M., \& Czochrowska, E. (2016). Dentes impactados: uma perspectiva interdisciplinar. Avanços na medicina clínica e experimental: órgão oficial Wroclaw Medical University, 25 (3), 575-585.

Khalaf, K., Al Shehadat, S., \& Murray, CA (2018). A Review of Supranumerary Teeth in the Premolar Region. Jornal internacional de odontologia, 2018.

Kumar, D. K., \& Gopal, K. S. (2013). An epidemiological study on supernumerary teeth: a survey on 5,000 people. Journal of clinical and diagnostic research: $J C D R, 7(7), 1504$.

Laganà, G., Lombardi, C. C, Franchi, L., \& Cozza, P. (2011). Tooth agenesis: dento-skeletal characteristics in subjects with ortodontic treatment need, 12 (1), $17-20$.

Luz, A. A., Faria, T. G. S., Catanoze, I. A., Ferreira, L. L., Bernabé, D. G., \& Miyahara, G. I. (2014). A importância do exame clínico criterioso no diagnóstico de lesões bucais. Revista de Odontologia da UNESP, 43(Especial), 0-0.

Lima, A. P. B., Costa, P. A., Barbosa, N. M. V., Almeida-Pedrin, R. R., Paranhos, L. R., \& de Almeida Cardoso, M. (2019). Segmented mechanics for traction of impacted maxillary canine: case report with a 3-year follow-up. Bioscience Journal, 35(1).

Marsi, G., Mengue, A. C., Bertini, F., Cabral, L. A. G., \& Almeida, J. D. (2009). Avaliação da importância do exame clínico para os alunos do curso de graduação da Faculdade de Odontologia de São José dos Campos-UNESP. Revista da ABENO, 9(1), 5-10.

Mohammed, A. K., Sravani, G., Vallappareddy, D., Rao, A. R., Qureshi, A., \& Prasad, A. N. (2020). Localization of Impacted Canines-A Comparative Study of Computed Tomography and Orthopantomography. Journal of medicine and life, 13(1), 56.

Ngan, P., Hornbrook, R., \& Weaver, B. (2005, September). Early timely management of ectopically erupting maxillary canines. In Seminars in Orthodontics (Vol. 11, No. 3, pp. 152-163). WB Saunders.

Oliveira, E. R., Alves, A. G. C., Marson, G. B. D. O., \& Hasse, P. N. (2015). Remoção cirúrgica de 5 dentes retidos em região anterior de mandíbula em posição ectópica. Revista UNINGÁ Review, 23(3).

Scully, A., Zhang, H., Kim-Berman, H., Benavides, E., Hardy, NC, \& Hu, JC (2020). Tratamento de dois casos de dentes supranumerários. Odontologia pediátrica, $42(1), 58-61$.

Stringhini Junior, E., Stang, B., \& Oliveira, L. B. (2015). Dentes supranumerários impactados: relato de caso clínico. Revista da Associacao Paulista de Cirurgioes Dentistas, 69(1), 89-94.

Stuani, V. D. T., Sant'Ana, A. C. P., Soares Junior, L. A. V., \& Santos, P. S. D. S. (2016). The relationship between periodontal disease and oral cancer. Revista Brasileira de Odontologia, 73(3), 218-222.

Vuchkova, J., \& Farah, C. S. (2010). Canine transmigration: comprehensive literature review and report of 4 new Australian cases. Oral Surgery, Oral Medicine, Oral Pathology, Oral Radiology, and Endodontology, 109(4), 46-53. 